\title{
Toward a unified genetic map of higher plants, transcending the monocot-dicot divergence
}

Andrew H. Paterson, Tien-Hung Lan, Kim P. Reischmann, Charlene Chang, Yann-Rong Lin, Sin-Chieh Liu, Mark D. Burow, Stanley P. Kowalski, Catherine S. Katsar, Terrye A. DelMonte, Kenneth A. Feldmann, Keith F. Schertz \& Jonathan E. Wendel

Nature Genetics 14, 380-382 (1996).

Due to a printing error, the lines for the chromosomes in Fig. 1 were omitted. A complete version is presented below.
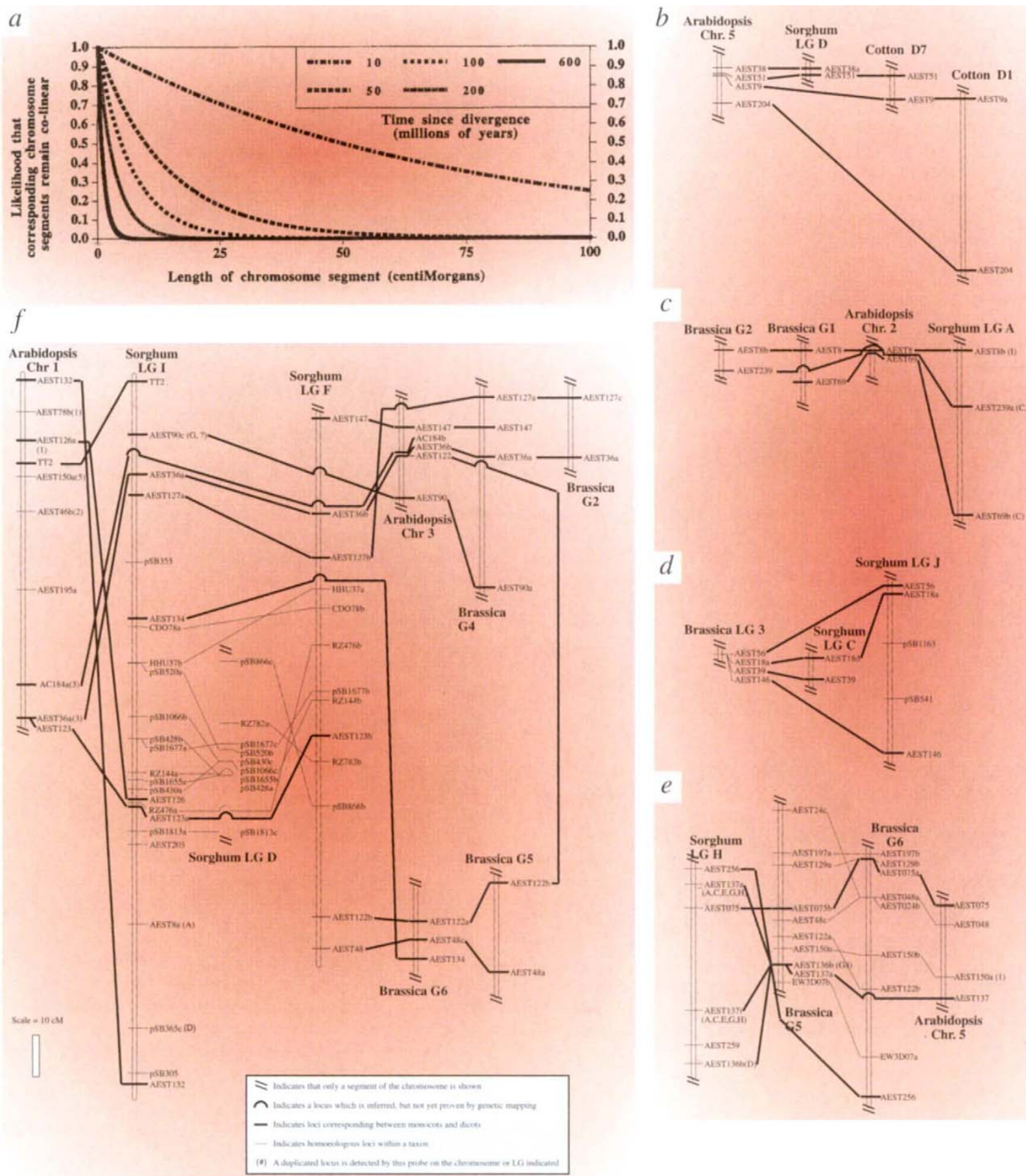